\title{
Modeling and Analysis of a Closed-Loop System for High-Q MEMS Accelerometer Sensor
}

\author{
Yalin Wang and Yongjun Yang \\ Hebei Semiconductor Research Institute, 050057 Shijiazhuang, China
}

\begin{abstract}
High-Q sensing element is desirable for high performance while makes the loop control a great challenge. This paper presents a closed-loop system for high-Q capacitive MEMS accelerometer which has achieved loop control effectively. The proportional-derivative(PD)control is developed in the system to improve the system stability. In addition, pulse width modulation (PWM) electrostatic force feedback is designed in the loop to overcome the nonlinearity. Furthermore, a sigma-delta $(\Sigma \Delta)$ modulator with noise shaping is built to realize digital output. System model is built in Matlab/Simulink. The simulation results indicate that equivalent $\mathrm{Q}$ value is reduced to 1.5 to ensure stability and responsiveness of the system. The effective number of bits of system output is 14.7 bits. The system nonlinearity is less than $5 \%$. The equivalent linear model including main noise factors is built, and then a complete theory of noise and linearity analysis is established to contribute to common MEMS accelerometer research.
\end{abstract}

\section{Introduction}

The capacitive microaccelerometer with high measurement precision, stable output, low temperature drift, is one of the most widely used MEMS sensors. High performance MEMS accelerometer with high-Q, low Brownian noise, are increasingly needed in oil exploration, inertial navigation and guidance, microgravity measurement in space, earthquake prediction and other fields.

Traditional MEMS accelerometer system research is concentrated in open-loop with analog output[1]. Recently more research on high-Q MEMS accelerometer is focused on the closed loop system, mainly divided into analog and digital closed-loop[2-4]. Closed-loop with analog output makes quality block movement near the equilibrium position improving the linearity[5]. But it is easily affected by the precision and noise of circuit meanwhile it is not conducive to the subsequent signal processing because of analog output. Another high-order $\Sigma \Delta$ loop system realizes low power consumption, low circuit complexity and digital output. But high order system lead to instability and coupling problem between mechanical and circuit[6]. This work will present a system which effectively reduces these unfavorable factors mentioned above. Moreover the theory on noise and linearity will contribute to the MEMS system research.

In this paper, we study a digital closed-loop system for high-Q MEMS accelerometer sensor which combines PD controller with $\Sigma \Delta$ modulator. The structure is organized as follows: Section 2 presents an electrical model of MEMS sensitive structure, and designs a PD control loop. Section 3 puts forward a new structure of $\Sigma \Delta$ modulator, and simulates the system model. Section 4 establishes the equivalent linear model, puts forward a theory of noise and linearity analysis. Finally, Section 5 concludes the paper and points out further work.

\section{MEMS model and PD control loop}

\subsection{The system-level design}

The system-level block diagram is shown in Fig.1.

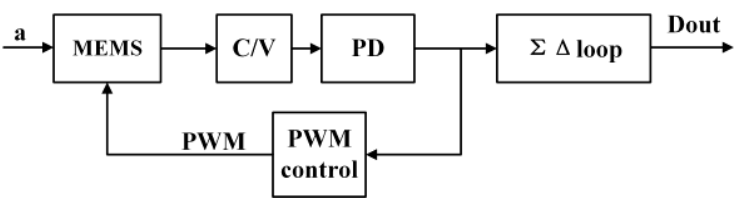

Figure 1. System-level block diagram

The system consists of MEMS mechanical structure, capacitor voltage conversion circuit, PD control loop with PWM feedback and $\Sigma \Delta$ modulator. The external acceleration signal causes a displacement of the MEMS sensitive structure's mass. The displacement causes the change of differential capacitance and converts to analog voltage. PD control loop ensures the stability and linearity of the system. Analog voltage is converted to digital signal by $\Sigma \Delta$ modulator and quantization noise is shaped to decrease the system noise level. 


\subsection{Electrical model and working principle of the MEMS sensitive structure}

Different from traditional sandwich structure, the MEMS sensitive structure studied in this paper is the comb type, as showed in Fig. 2.

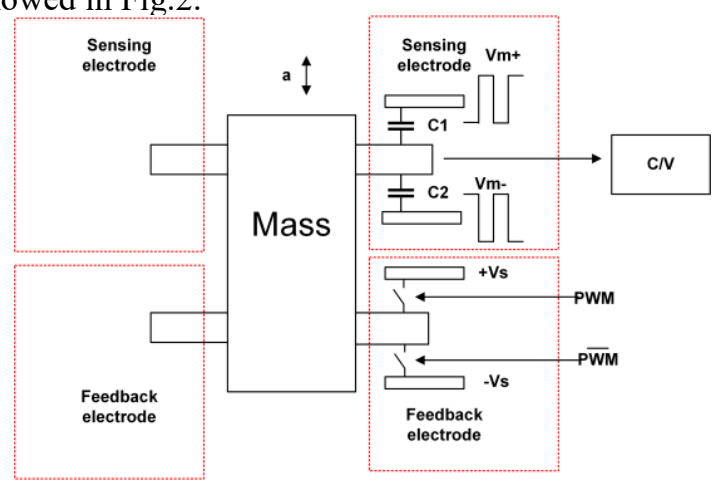

Figure 2. The simplified model of MEMS sensor

It consists of movable mass and fixed electrode with bilateral symmetrical structure. Sensing electrode is connected to front-end circuit realizing capacitor-voltage conversion $(\mathrm{C} / \mathrm{V})$. Feedback electrode is connected to feedback voltage. The device of MEMS accelerometer can be modeled as a 2nd order spring-damping system with external acceleration input. The equation of motion can be written as:

$$
m \frac{d^{2} x}{d t^{2}}+b \frac{d x}{d t}+k x=m a(t)
$$

Where $\mathrm{m}$ is the proof mass, $\mathrm{x}$ is the displacement of the mass, $b$ is the damping coefficient, $\mathrm{k}$ is the spring coefficient. The acceleration-to-displacement transfer function is written as:

$$
M(s)=\frac{X(s)}{A(s)}=\frac{1}{s^{2}+s \frac{b}{m}+\frac{k}{m}}=\frac{1}{s^{2}+s \frac{\omega_{0}}{Q}+\omega_{0}^{2}}
$$

Where $\omega 0$ is the resonant frequency, and $\mathrm{Q}$ is the quality factor.

The mass moves near the balance position to ensure the system work in a linear state[7]. The PD closed-loop as follows will realize this condition. When PD control is added,the system transfer function is given as:

$$
M^{\prime}(s)=\frac{M(s) K_{C / V}}{1+M(s) K_{C / V} K f b\left(K p+K_{d} s\right)}
$$

Where $K_{C / V}$ is the capacitance to voltage conversion factor, $K_{f b}$ is the voltage to force conversion factor, $K_{p}$ is proportional coefficient and $K_{d}$ is differential coefficient.

The MEMS electrical model with PD control is shown in Fig.3. The equivalent $\mathrm{Q}\left(Q_{E}\right)$ and resonant frequency $\omega$ of system is given as:

$$
\begin{gathered}
Q_{E}=\frac{\sqrt{m\left(k+K p K f b K_{C / V}\right.}}{b+K d K f b K_{C / V}} \\
\omega=\sqrt{\frac{k+K p K f b K_{C / V}}{m}}
\end{gathered}
$$

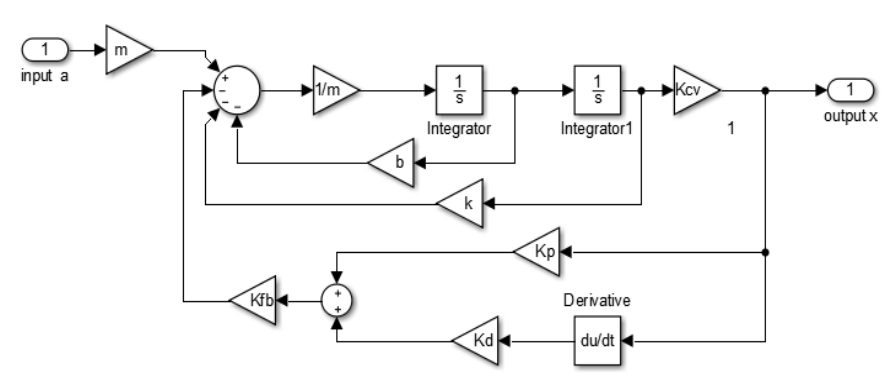

Figure 3. The Simulink model of closed-loop MEMS with PD control

The optimal system is presented by setting appropriate parameters. According to the bode diagram (Fig.4) and square input response(Fig.5), the $Q_{E}$ is 1.5 while open loop's Q is 50.2 , and $\omega$ is up to $2200 \mathrm{~Hz}$ while $\omega_{0}$ is $1050 \mathrm{~Hz}$. The PD control improved the stability by reducing the quality factor and broadened bandwidth of system.

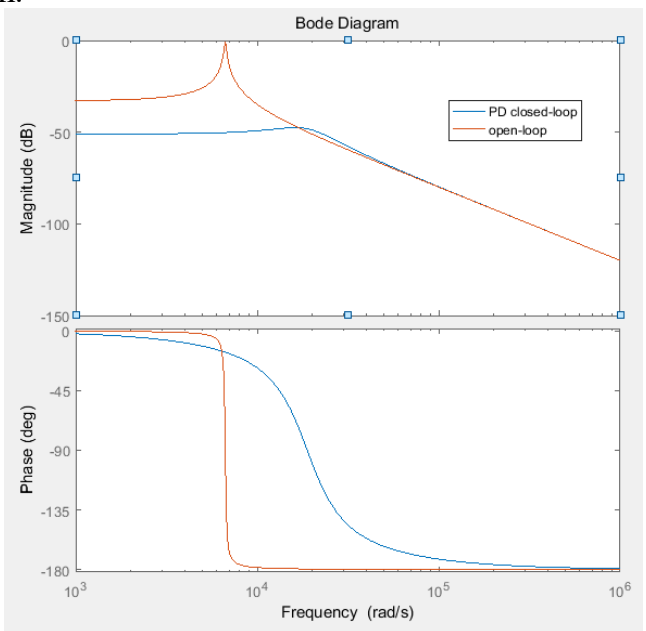

Figure 4. The system bode diagram

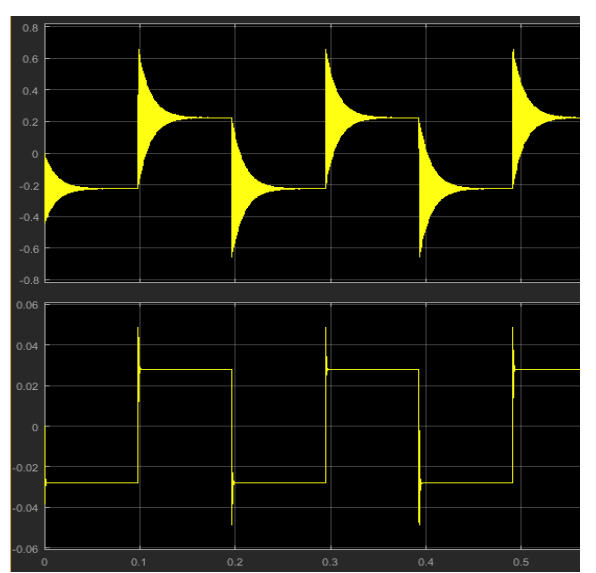

Figure 5. The system square input response graph

\section{$3 \Sigma \Delta$ modulator and system simulation}

The single loop $\Sigma \Delta$ modulators are sorted into two typical architectures called feed-forward (FF) and multiplefeedback (MF) architecture. The FF architecture has definite zeros and higher low-frequency gain than MF to achieve high linearity. But it has worse stability than MF. 
Meanwhile there are adjustable poles in the MF architecture to ensure stability, but it is poor in linearity[8]. Based on common $\Sigma \Delta$ modulators [9], the architecture in this paper as shown in Fig. 6, combined the FF with the MF with both comprehensive advantage: stability and linearity. There is phase delay in the system(Fig.5), the front-end phase compensator for $\Sigma \Delta$ modulator is necessary.

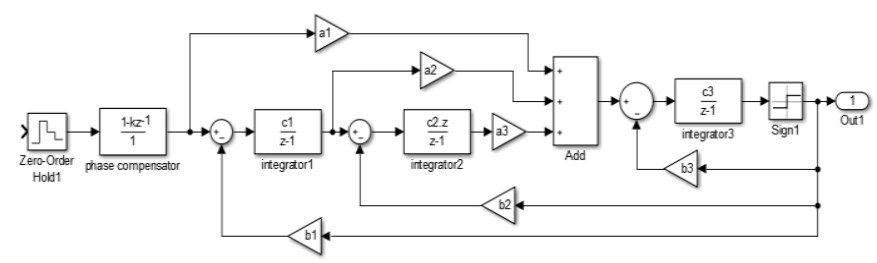

Figure 6. Architecture of the $\Sigma \Delta$ modulator

Where $\mathrm{k}=0.25$ to provide appropriate phase compensation. The optimal value of other parameters are determined by the scanning parameter method: $a 1=0.4$, $\mathrm{a} 2=0.3, \quad \mathrm{a} 3=0.3, \quad \mathrm{~b} 1=0.3, \quad \mathrm{~b} 2=0.3, \quad \mathrm{~b} 3=0.5$, $\mathrm{c} 1=0.5, \mathrm{c} 2=0.3, \mathrm{c} 3=0.4$. The transfer function is given as:

$$
H=\frac{0.16 z^{4}-0.402 z^{3}+0.3673 z^{2}-0.1387 z+0.016}{z^{5}-3.589 z^{4}+4.844 z^{3}-2.908 z^{2}+0.6544 z}
$$

When input signal is a sine wave with a magnitude of 10 and a frequency of $80 \mathrm{~Hz}$, oversampling ratio is 256 , the simulation results are showed in Fig. 7.

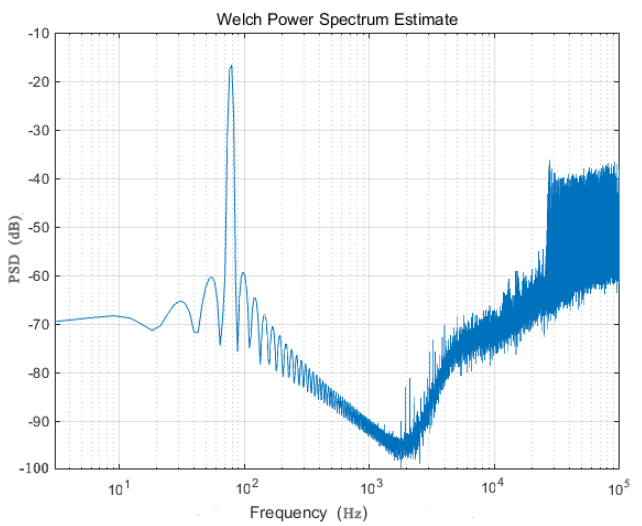

Figure 7. Output signal power spectral density

The simulation results show that the signal-to-noise ratio(SNR) is $90 \mathrm{~dB}$, the effective number of bits is 14.7 bits.

\section{Linearity and noise analysis}

\subsection{Linearity analysis}

The total nonlinearity of a closed-loop system is affected by the both forward signal path and feedback path[10]. Because the displacement is near the balance place by using PD control, the forward path nonlinearity is significantly depressed. The feedback path nonlinearity will dominate. Nonlinearity in feedback path is mainly composed of two parts: the mismatch between the actuation capacitors; and the non-zero proof mass displacement due to the finite loop gain. In [10],feedback force is given as:

$$
F_{f b}\left(V_{f b}\right)=\frac{T_{f b}}{T s}\left[\frac{\varepsilon A 1\left(V p-V_{f b}\right)^{2}}{2\left(d_{2}+x\right)^{2}}-\frac{\varepsilon A 1\left(V_{f b}-V n\right)^{2}}{2\left(d_{1}-x\right)^{2}}\right]
$$

The square law relationship between the electrostatic feedback force and the applied voltage leads to relatively poor nonlinearity up to several percent. In this work, time-control(pulse width modulation force feedback) rather than traditional voltage-control actuation is developed. The PWM control principle is shown in Fig.8.

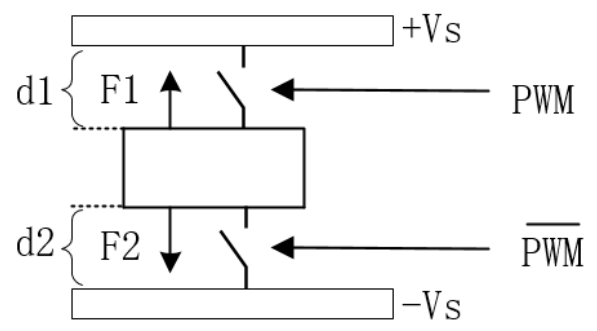

Figure 8. PWM control principle diagram

PWM signal controls the switch between movable mass and electrode with the voltage(+Vs\&-Vs). The feedback force $F_{f b}$ is proportional to the pulse width of the PWM signal $(\Phi)$. The feedback force is given as:

$$
F_{f b}=F 1-F 2=\frac{T_{S}-\Phi}{T_{S}} F 1-\frac{\Phi}{T_{S}} F 2
$$

Where $T_{S}$ is the sampling time, $F 1, F 2$ is the electrostatic force as follows:

$$
\begin{gathered}
F 1=\frac{\varepsilon \varepsilon_{0} A\left(-V_{s}-V s\right)^{2}}{2 d_{1}{ }^{2}} \\
F 2=\frac{\varepsilon \varepsilon_{0} A(V s+V s)^{2}}{2 d_{2}{ }^{2}}
\end{gathered}
$$

Where $\varepsilon_{0}$ is the dielectric constant in a vacuum, $\varepsilon$ is the dielectric constant between mass and plate electrode, $d_{1}$ is the distance between upper plate electrode and $d_{2}$ is the distance between under plate electrode.

Equation (8) (10) shows that mismatch between the actuation capacitors $(F 1 \neq F 2)$ in balance state is no longer the system nonlinearity factor, while displacement will still lead to the nonlinearity, thus an applicable loop gain is needed for linearity. A verilog-A model of the MEMS accelerometer was developed in Cadence. The nonlinearity (NL) VS mismatch and the loop gain is given as figure 9 .

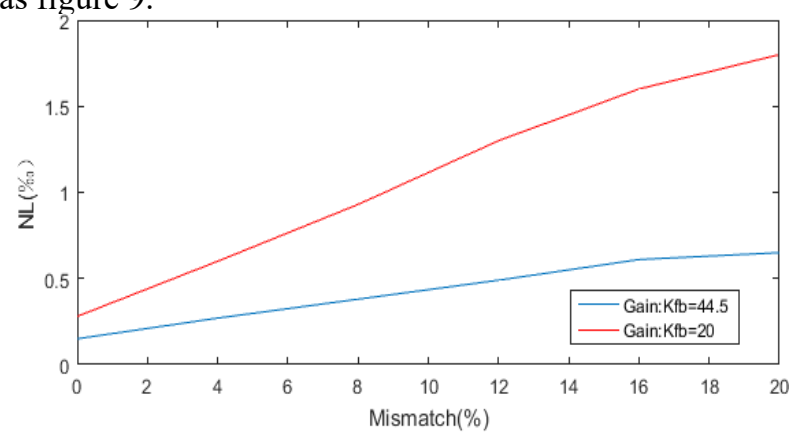

Figure 9. Nonlinearity (NL) VS mismatch and loop gain 
The simulation result indicates that mismatch increases to 20 , NL is changed about $0.4 \%$ when the loop gain is 44.5 .

\subsection{Noise analysis}

The system noise consists of mechanical noise(Brownian noise), charge amplifier noise, quantization noise, integrator noise. Based on noise factors, the equivalent linear model is given as Fig.10

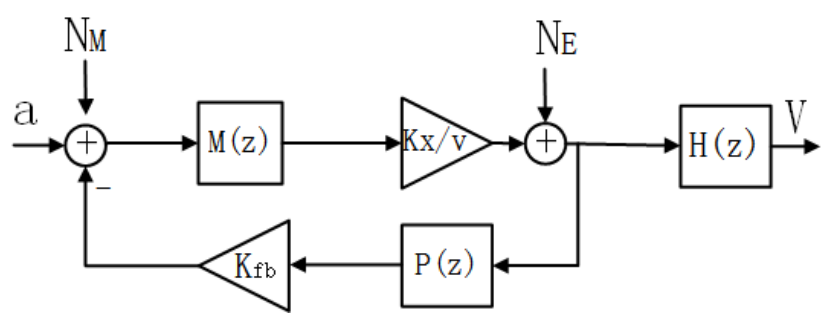

Figure 10. System equivalent linear model with noise factors

Where NM is mechanical noise, NA is charge amplifier noise, $\mathrm{P}(\mathrm{z})$ is $\mathrm{PD}$ controller transfer function. $\mathrm{H}(\mathrm{z})$ is $\Sigma \Delta$ modulator, the noise model is showed in Fig. 11 .

It is helpful to get the noise transfer function to evaluate the noise impact on the system noise

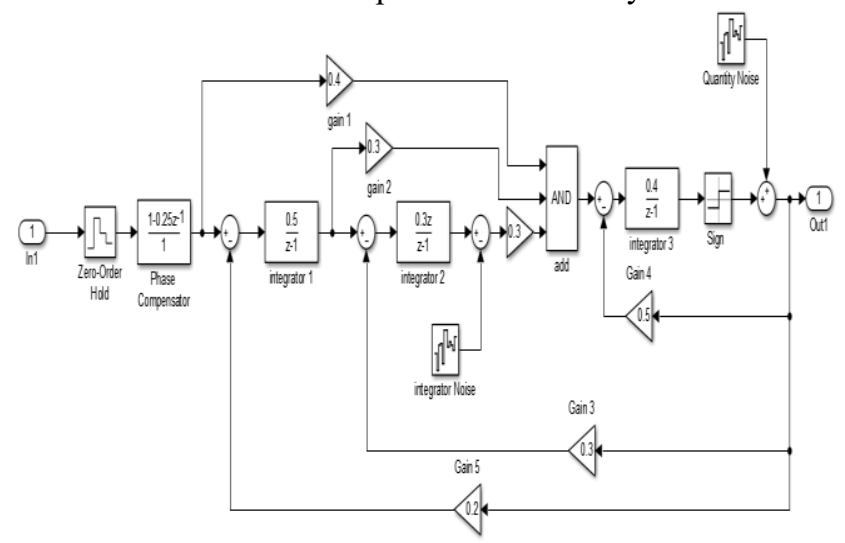

Figure 11. $\Sigma \Delta$ modulator structure with noise factors

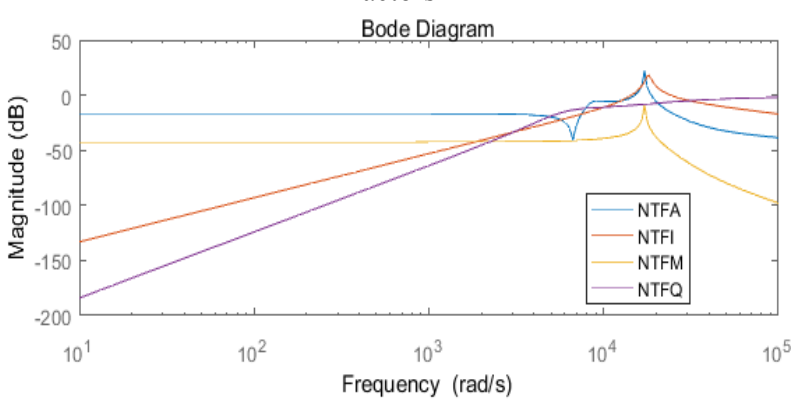

Figure 12. Simulated frequency responses of the noise transfer functions

Where $K_{b}$ is Boltzmann constant $\left(1.38 \times 10^{-23} J / K\right)$, T is Kelvin temperature, $\mathrm{m}$ is the proof mass, $\mathrm{b}$ is the damping factor. Because of vacuum packaging, $b$ is small, leading to small mechanical noise. The mechanical noise of performance. The noise transfer function (NTF) can be written as:

$$
\begin{gathered}
N T F M=\frac{K_{X / V} M(z) H(z)}{1+K_{f b} K_{X / V} M(z) P(z)} \\
N T F A=\frac{H(z)}{1+K_{X / V} K_{f b} M(z) P(z)} \\
N T F I=\frac{0.12 z^{3}-0.336 z^{2}+0.312 z-0.096}{z^{4}-3.589 z^{3}+4.489 z^{2}-2.918 z+0.6587} \\
N T F Q=\frac{z^{3}-3 z^{2}+3 z-1}{z^{3}-3.4 z^{2}+3.782 z-1.384}
\end{gathered}
$$

where $N T F M, N T F A, N T F I, N T F Q$ respectively are the transfer function of mechanical noise, charge amplifier noise, integrator noise and quantization noise.

The frequency responses of the noise transfer functions are plotted in Fig .12.

At low frequency $(\mathrm{f}<10000 \mathrm{rad} / \mathrm{s})$ as shown in Fig. 12, $N T F A>N T F M>N T F I>N T F Q$, thus charge amplifier noise and mechanical noise are the main noise source. Mechanical noise is caused by the interaction of the air molecules surrounding called Brownian movement.It is a white noise, and it's power spectrum is defined as follows :

$$
a_{n}^{2}=\frac{4 K_{b} T b}{m^{2}}
$$

MEMS researched in this paper is nearly $20 \mathrm{ng} / \sqrt{\mathrm{Hz}}$. Compared with other noise, it is almost negligible. The charge amplifier noise becomes dominant noise source.

Figure 13 describes the front-end interface circuit including charge amplifier and MEMS sensor. Vm is the reference voltage of MEMS sensor, Vcom is the common mode voltage of the amplifier, and $\mathrm{Vx}$ is the output of amplifier. When an acceleration comes, the mass will change its displacement, and the charge amplifier will convert the capacitance variation to voltage. When mass is balanced without extra acceleration:

$$
C 0=\frac{\varepsilon \varepsilon_{0} A}{d_{0}}
$$

When input acceleration lead to a displacement $\mathrm{x}$,

$$
\begin{gathered}
C 1=\frac{\varepsilon \varepsilon 0 A}{d_{0}-x} \\
C 1=\frac{\varepsilon \varepsilon 0 A}{d_{0}+x} \\
\Delta C=C 1-C 2=C 0\left(\frac{d_{0}}{d_{0}-x}-\frac{d_{0}}{d_{0}+x}\right) \\
=C 0\left[\sum_{n=0}^{\infty}\left(\frac{x}{d_{0}}\right)^{n}-\sum_{n=0}^{\infty}\left(-\frac{x}{d_{0}}\right)^{n}\right] \\
=2 C 0\left[\frac{x}{d_{0}}+\left(\frac{x}{d_{0}}\right)^{3}+\left(\frac{x}{d_{0}}\right)^{5}+\ldots\right. \\
\approx 2 C 0 \frac{x}{d_{0}},\left(x<<d_{0}\right)
\end{gathered}
$$

In $\phi 1$ and $\phi 2$, the charges stored in different capacitors are written as:

$$
Q_{C 1}(\phi 1)=\left(V_{m}-V_{c o m}\right) * C 1
$$




$$
\begin{aligned}
& Q_{C 2}(\phi 1)=\left(V_{m}+V_{c o m}\right) * C 2 \\
& Q_{C f}(\phi 1)=V_{c o m} * C f \\
& Q_{C 1}(\varphi 2)=\left(-V_{m}-V_{c o m}\right) * C 1 \\
& Q_{C 2}(\varphi 2)=\left(V_{c o m}-V_{m}\right) * C 2 \\
& Q_{C f}(\phi 2)=\left(V_{c o m}-V_{x}\right) * C f
\end{aligned}
$$

When ph1 changes to ph2,s charges change of the feedback capacitor $C f$ is given as:

$$
\Delta Q_{f}=\Delta Q_{f}(\phi 1)-\Delta Q_{f}(\phi 2)=2 V_{m} *(C 1-C 2)=V_{x} C f
$$

Thus

$$
V_{x}=2 V_{m} \frac{C 1-C 2}{C f}=4 V_{m} C 0 \frac{x}{d_{0} * C f}
$$

The charge amplifier noise mainly consists of operation amplifier noise, flicker noise, reference voltage noise and switch noise[11]. The flicker noise which be reduced by correlated double sampling technique will no longer be the main noise in this paper. To calculate the noise, the charge amplifier schematic can be simplified as Fig. 14.

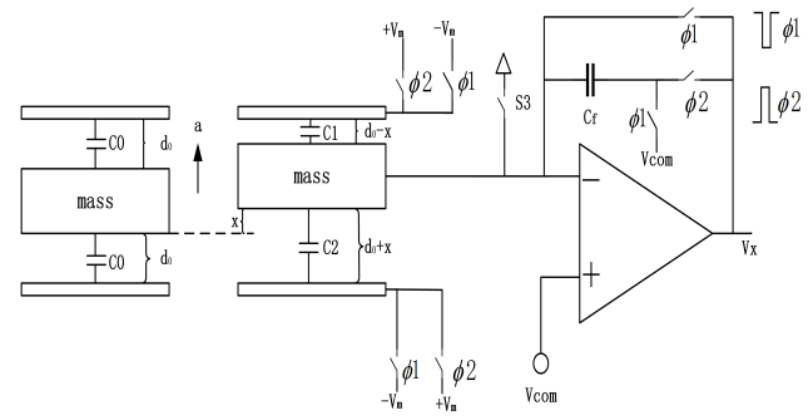

Figure 13. Front-end interface circuit

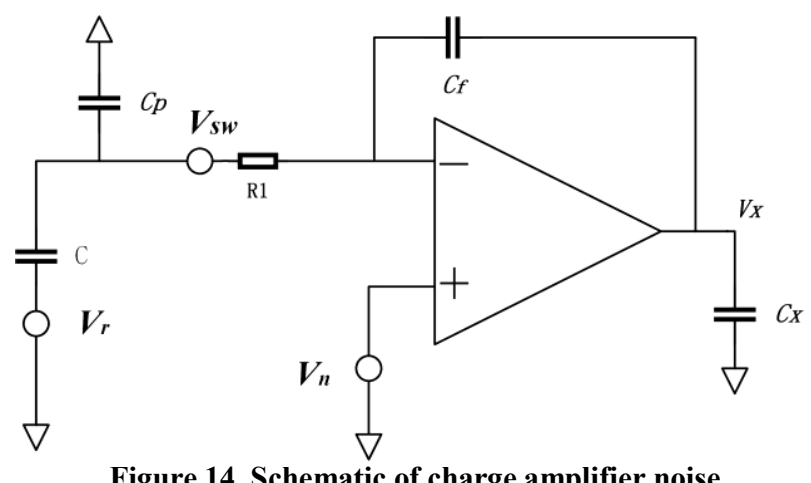

Figure 14. Schematic of charge amplifier noise

The operation amplifier noise $\left(V_{n}\right)$, the switch noise $\left(V_{s w}\right)$ and the reference voltage noise $\left(V_{r}\right)$ are written as:

$$
\begin{gathered}
V_{n}=\sqrt{\frac{16 K_{b} T(C+C p)}{3 f_{s} C x C f}} \\
V_{s w}=\frac{1}{C f} \sqrt{\frac{2 K_{b} T R 1 g_{m} C}{f_{s}}} \\
V_{r}=\frac{V_{r f}}{C f} \sqrt{\frac{g_{m} C}{f_{S}}}
\end{gathered}
$$

Where $K_{b}$ is Boltzmann constant $\left(1.38 \times 10^{-23} J / K\right), f_{S}$ is the sampling frequency, $C x$ is the load capacitance, $C f$ is the feedback capacitance, $C$ is the sensing capacitance, and $C p$ is the parasitic capacitance, $V_{r f}$ is the reference voltage noise at low-frequency (assumed to be white noise with an in-band spectral density of $8 \mathrm{nV} / \sqrt{\mathrm{Hz}}$ ), $R 1$ is the equivalent resistance of the switch, and $g_{m}$ is the trans-conductance of the input transistor of the charge amplifier.

According to the design parameters, three main types of noise are calculated. In this paper, $f_{S}=256 \mathrm{KHz}, C x=10 P F$, $C f=12 P F, C=280 P F, C p=15 P F, g_{m}=3 m s, R 1=180 \Omega$. Thus, $V_{n}=0.4253 \mu \mathrm{g} / \sqrt{\mathrm{Hz}}, V_{S W}=0.1702 \mu \mathrm{g} / \sqrt{\mathrm{Hz}}, V_{r}=1.2076 \mu \mathrm{g} / \sqrt{\mathrm{Hz}}$. The switch noise has less impact on system than operation amplifier noise and reference voltage noise. The reference voltage noise should be reduced firstly in the design of system-level low noise optimization.

\section{Conclusions}

In this paper, we establish a closed-loop system for highQ MEMS accelerometer sensor, clarify the equivalent electrical model of MEMS accelerometer sensor and design a PD closed-loop with proper coefficient to improve the stability of the system. Then we design a new structure of sigma-delta modulator with good stability and high linearity to realize digital output. Finally, we put forward a linearity analysis theory, use PWM technology in $\mathrm{q}$ feedback loop to improve linearity and build a compete noise analysis theory which will provide the theoretical basis for the latter circuit design and MEMS accelerometer sensor system research. The future research work includes the low-power interface circuit design and function test after fabrication.

\section{References}

1. B Borovic1, et al, "Open-loop versus closed-loop control of MEMS devices choices and issues", Journal of Micromechanics and micorengineering, pp1917 - 1924,2005

2. Zhaohui Song, Teng Sun, and Jian Wu, "System-level simulation and implementation for a high Q capacitive accelerometer with PD feedback compensation", Microsyst Technol, vol. pp. 22332240, 2015.

3. Yin Liang, et al, "High resolution interface circuit for closed-loop accelerometer", Journal of Semiconductors, vol. 32, pp.(045005)1-8, 2011

4. Yuntao Liu et al, "Stability Analysis in High-Order Electromechanical Sigma-Delta Accelerometer", Key Engineering Materials, vol 483, pp 716-720, 2011

5. Bin Wang, et al, "Model of Closed-loop Detection System for Capacitive MEMS Accelerometer and its IC Realization", Advanced Materials Research, vol 468, pp 2170-2175, 2012

6. Shaodong Chen, Honglin $\mathrm{Xu}$, "Design analysis of a high-Q micromechanical capacitive accelerometer 
system”, IEICE Electronics Express, vol.14, pp.1-6, 2017

7. Mingjun MA, Zhong He, and Hui Jie, "A combined modulated feedback and temperature compensation approach to improve bias drift of a closed-loop MEMS capacitive accelerometer", Frontiers of Information Technology \& Electronic Engineering, vol.16, pp.497-510, 2015

8. Zhang Meimei, Quan Haiyang and Zhang Fuqiang, "Comparisons of Feed-Forward and MultipleFeedback Sigma-Delta Modulators for MEMS Accelerometers", MATEC Web of Conferences, 08003,2016
9. Zhimin Li, Terri S. Fiez, "A 14 Bit Continuous-Time Delta-Sigma A/D”, IEEE Journal of Solid-State Circuits, vol. 42, pp.1873-1883, 2007.

10. Y.F.Dong, Kraft.M, "Force feedback linearization for higher-order electromechanical sigma-delta modulators, Journal of Micromechanics and micorengineering”, vol.16, pp.54-60, 2006

11. H. Kulah, J. Chae, and K. Najafi, "Noise analysis and characterization of a sigma-delta capacitive microaccelerometer", IEEE Journal of Solid-State Circuits, vol.41, pp.352 - 361, 2006 